\title{
Cell Wall Turnover During Myxospore Formation in Myxococcus xanthus
}

\author{
By HILARY DAWSON AND M. V. JONES \\ Department of Microbiology, University of Liverpool, Life Sciences Building, \\ P.O. Box 147, Liverpool L69 3BX
}

(Received 27 September 1978)

\begin{abstract}
Wall turnover in Myxococcus xanthus has been studied by measuring the incorporation and release of meso-diamino $\left[{ }^{14} \mathrm{C}\right]$ pimelic acid. The rate of turnover was low in vegetatively growing organisms. Following the addition of $0.5 \mathrm{M}$-glycerol to induce myxospore formation in organisms that had been labelled for one generation in the absence of glycerol, increased rates of incorporation and release were observed both before and during cellular morphogenesis. Chloramphenicol inhibited glycerol-induced myxospore formation, but only partially blocked the release of meso-diaminopimelic acid. A mutant, non-inducible by glycerol, was isolated and was found to show no increased autolytic activity in the presence of glycerol. Both parent and mutant strains could be induced to form myxospores by $17 \mathrm{~mm}$ phenethyl alcohol, but with both strains there was only a slight increase in meso-diaminopimelic acid release following induction.
\end{abstract}

\section{INTRODUCTION}

The fruiting myxobacterium, Myxococcus xanthus, grows in liquid media containing protein hydrolysate with a generation time of 4 to $5 \mathrm{~h}$. When deprived of nutrients whilst growing on solid media, the cells aggregate and construct multicellular fruiting bodies within which a proportion of the rod-shaped cells develop into spherical resting cells or myxospores. This normal developmental cycle can be short-circuited by the addition of a variety of hydroxylated compounds, e.g. glycerol or phenethyl alcohol, to exponentially growing organisms which then convert directly into myxospores (Dworkin \& Gibson, 1964; Sadler \& Dworkin, 1966). This provides an interesting system for studying the control of cellular morphogenesis in a Gram-negative bacterium.

The mechanism by which the various inducing compounds act is unknown. Following the addition of $0.5 \mathrm{M}$-glycerol to a growing culture there is a rapid and synchronous conversion of the rod-shaped cells to spheres (Dworkin \& Gibson, 1964). This shape change is preceded by greatly increased cellular autolytic activity (Kottel \& White, 1974). In a mutant in which the shape change occurred $8 \mathrm{~h}$ after glycerol addition, the increased autolytic activity was similarly delayed (Kottel $\&$ White, 1974). No major differences in composition between the peptidoglycan of vegetative organisms and myxospores have been found, but transient changes in the degree of cross-linking were reported (White et al., 1968). The shape change could therefore involve modification of the existing wall or replacement with peptidoglycan with an altered structure.

We have been investigating this problem by following the incorporation and retention of meso-diamino $\left[{ }^{14} \mathrm{C}\right]$ pimelic acid in peptidoglycan during the formation of myxospores by $M$. xanthus FB and in a mutant that is unable to form myxospores in the presence of glycerol. A preliminary report of part of this work has been published (Dawson \& Jones, 1978). 


\section{METHODS}

Organism and growth conditions. Myxococcus xanthus FB (NCIB 9412) was grown aerobically at $30^{\circ} \mathrm{C}$ in $1 \%(\mathrm{w} / \mathrm{v})$ Bacto-Casitone (Difco) containing $8 \mathrm{~mm}-\mathrm{MgSO}_{4}$. Stock cultures were maintained on the same medium solidified with $1.5 \%(\mathrm{w} / \mathrm{v})$ Davis agar. Myxospore formation was induced in liquid cultures by the addition of glycerol at a final concentration of $0.5 \mathrm{M}$ or phenethyl alcohol at a final concentration of $17 \mathrm{~mm}$ (Sadler \& Dworkin, 1966).

Isolation of a glycerol-insensitive mutant. A spontaneous mutant which cannot be induced to form myxospores by the addition of glycerol was isolated and maintained on agar medium containing $1 \%$ (w/v) BactoCasitone, $0.5 \mathrm{M}$-glycerol and $8 \mathrm{~mm}-\mathrm{MgSO}_{4}$.

Measurement of wall turnover. Peptidoglycan was labelled by incubation of exponentially growing organisms for $4 \mathrm{~h}$ with (DL + meso)-2,6-diamino[1,7-14 $\mathrm{C}]$ pimelic acid dihydrochloride $\left(50 \mathrm{mCi} \mathrm{mmol}{ }^{-1}, 0 \cdot 1 \mu \mathrm{Ci} \mathrm{m}{ }^{-1}\right.$; The Radiochemical Centre, Amersham). After labelling, the myxococci were pelleted by centrifugation $(12000 \mathrm{~g}, 10 \mathrm{~min})$, washed in distilled water, resuspended in fresh growth medium at a density of approximately $2 \times 10^{8}$ organisms $\mathrm{ml}^{-1}$ and incubated with shaking at $30^{\circ} \mathrm{C}$. At intervals, samples $(0 \cdot 3 \mathrm{ml})$ were taken and filtered under vacuum through $24 \mathrm{~mm}$ Whatman GF/C discs. Filters were washed twice with $2 \mathrm{ml}$ $8 \mathrm{mM}^{-\mathrm{MgSO}_{4}}$ and once with $2 \mathrm{ml} 10 \%(\mathrm{w} / \mathrm{v})$ trichloroacetic acid. After drying at $80{ }^{\circ} \mathrm{C}$, the filters were counted for radioactivity in toluene containing 2,5-diphenyloxazole $\left(7 \mathrm{~g} \mathrm{l}^{-1}\right)$ using an Intertechnique SL30 liquid scintillation spectrophotometer. In some experiments turnover was measured by determining the radioactivity released into the medium. Culture samples $(0.2 \mathrm{ml})$ were taken and the organisms were pelleted in $30 \mathrm{~s}$ using a Unipan 320 microcentrifuge. Supernatant samples $(75 \mu \mathrm{l})$ were counted in toluene/Triton scintillation fluid ( $500 \mathrm{ml}$ Triton X-100; $7 \mathrm{~g}$ 2,5-diphenyloxazole; 11 toluene).

A wall fraction was prepared from exponentially growing organisms by treatment with hot sodium dodecyl sulphate by the method of White et al. (1968).

Uptake and incorporation of meso-diaminopimelic acid. Uptake of the amino acid was measured by adding meso-diamino $\left[{ }^{14} \mathrm{C}\right]$ pimelic acid (final activity of $0 \cdot 1 \mu \mathrm{Ci} \mathrm{ml}^{-1}$ ) to a culture containing approximately $2 \times 10^{8}$ organisms $\mathrm{ml}^{-1}$. At intervals, samples were taken, filtered, and the filter discs were washed twice with $2 \mathrm{ml}$ $8 \mathrm{~mm}^{-\mathrm{MgSO}_{4}}$. The radioactivity of the dried discs was determined as described above.

Incorporation of meso-diaminopimelic acid into walls was examined by pulse-labelling the organisms. Samples $(2 \mathrm{ml})$ were removed at intervals from a master culture and incubated at $30^{\circ} \mathrm{C}$ for 20 min with the labelled amino acid. After pulsing, $0.4 \mathrm{ml}$ portions were transferred to separate tubes containing $1 \mathrm{ml} 10 \%$ (w/v) trichloroacetic acid (TCA). After $30 \mathrm{~min}$ at room temperature the TCA-insoluble material was removed by filtration and the incorporated radioactivity was determined on the washed and dried filters.

Measurement of autolytic activity. The method was a modification of that used by Kottel \& White (1974). An exponentially growing culture was centrifuged and the myxococci were resuspended in fresh growth medium at a density of approximately $2 \times 10^{8}$ organisms $\mathrm{ml}^{\mathbf{- 1}}$. After addition of glycerol (to $0.5 \mathrm{M}$ final concentration), samples $(0.5 \mathrm{ml})$ were removed at intervals and added to $5 \mathrm{ml}$ distilled water prewarmed to $50{ }^{\circ} \mathrm{C}$. The absorbance at $550 \mathrm{~nm}$ was measured immediately and after $15 \mathrm{~min}$ incubation at $50{ }^{\circ} \mathrm{C}$. The percentage decrease in absorbance was taken as a measure of autolytic activity.

\section{RESULTS}

\section{Wall turnover}

To establish that incorporated meso-diaminopimelic acid was located in the peptidoglycan, a sodium dodecyl sulphate-insoluble fraction was prepared from labelled organisms. Almost all $(93 \%)$ of the incorporated activity was recovered in this fraction. Furthermore, $89 \%$ of this radioactivity was solubilized by overnight incubation of the sodium dodecyl sulphate-insoluble material with lysozyme $\left(100 \mu \mathrm{g} \mathrm{ml}^{-1}\right)$.

A slow rate of wall turnover, as measured by the retention of meso-diaminopimelic acid in organisms prelabelled for one generation, was observed during vegetative growth. About $2.8 \%$ of the radioactivity was released $\mathrm{h}^{-1}$ (Fig. 1). In contrast, significant release of radioactivity occurred when myxospore formation was induced by the addition of glycerol. The rate of release increased quickly after adding the inducer and approximately $60 \%$ of the label was lost in the first $2.5 \mathrm{~h}$ during which the shape change occurred (Fig. 1). When organisms were labelled with $\left[{ }^{14} \mathrm{C}\right]$ leucine, an amino acid not found in $M$. xanthus peptidoglycan (White et al., 1968), only low levels of release were observed in the presence or absence of glycerol. 


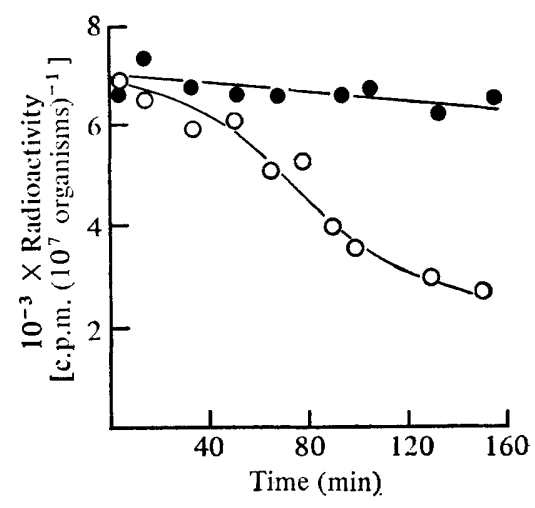

Fig. 1. Effect of glycerol on the release of meso-diamino[ $\left.{ }^{14} \mathrm{C}\right]$ pimelic acid from $M y x o c o c c u s$ xanthus. Organisms were prelabelled with amino acid, washed and resuspended in fresh medium. Loss of amino acid was measured by determining the radioactivity remaining in organisms incubated with (O) or without (O) $0.5 \mathrm{M}$-glycerol.

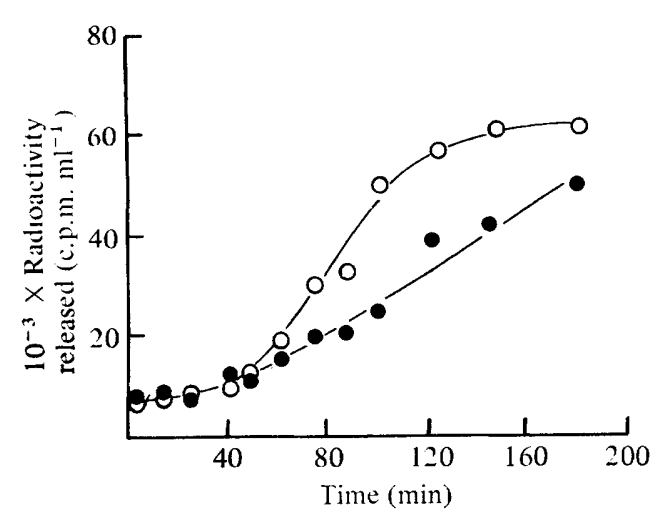

Fig. 2

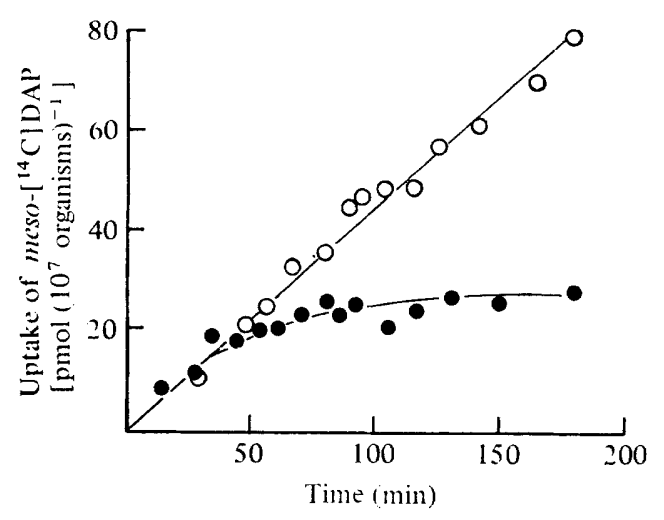

Fig. 3

Fig. 2. Effect of chloramphenicol on the release of radioactivity from Myxococcus xanthus into the medium in the presence of glycerol. Organisms were prelabelled with amino acid, washed and resuspended in fresh medium. Release of meso-diamino $\left[{ }^{14} \mathrm{C}\right]$ pimelic acid into the medium was determined by centrifuging the cultures and assaying the supernatant. Organisms were incubated in the presence of $0.5 \mathrm{M}$-glycerol with $(\Theta)$ or without $(O)$ chloramphenicol $\left(200 \mu \mathrm{g} \mathrm{ml}^{-1}\right)$.

Fig. 3. Effect of glycerol on the uptake of meso-diamino $\left[{ }^{14} \mathrm{C}\right]$ pimelic acid (meso- $\left[{ }^{14} \mathrm{C}\right] \mathrm{DAP}$ ) into Myxococcus xanthus incubated in growth medium with $(O)$ or without $(O) 0.5 \mathrm{M}$-glycerol.

Myxococcus xanthus clumps after glycerol addition, and turnover was found to be more accurately measured by determining the radioactivity released into the medium rather than that retained by the organisms. An apparently high level of released radioactivity at $t_{0}$ was probably due to both release of pool material and an inadequate washing procedure. However, more rigorous washing was avoided as this resulted in some cell lysis and delayed morphogenesis. The rate of label release increased sharply around 40 to $50 \mathrm{~min}$ after glycerol addition (Fig. 2). This just preceded the period of cellular morphogenesis. When chloramphenicol was added with glycerol, there was no shape change and myxospores were not formed. In the presence of both glycerol and chloramphenicol some increased release of label was observed but the rate after $40 \mathrm{~min}$ was substantially less than that occurring in the absence of the inhibitor (Fig. 2). 


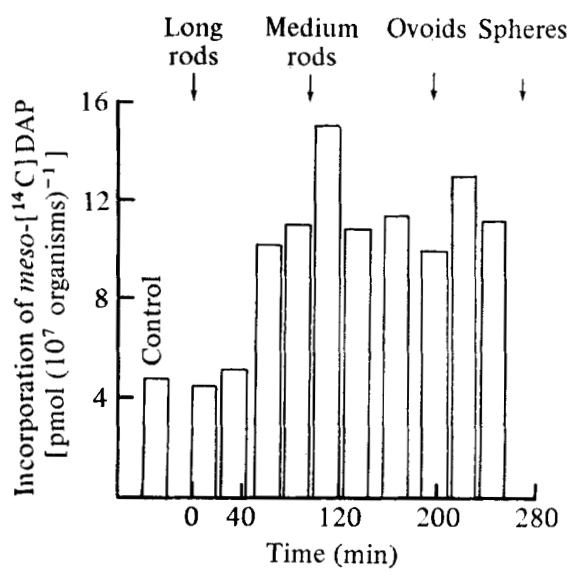

Fig. 4. Incorporation of meso-diamino $\left[{ }^{14} \mathrm{C}\right]$ pimelic acid (meso- $\left[{ }^{14} \mathrm{C}\right] \mathrm{DAP}$ ) into Myxococcus xanthus during glycerol-induced myxospore formation. The amount of ${ }^{14} \mathrm{C}$-labelled material incorporated into TCA-insoluble material during $20 \mathrm{~min}$ pulses was determined both before and after induction with $0.5 \mathrm{M}$-glycerol at time zero.

\section{Uptake and incorporation of meso-diaminopimelic acid}

Meso-diaminopimelic acid was rapidly taken up by growing $M$. xanthus (Fig. 3), but 50 to $60 \mathrm{~min}$ after adding glycerol, uptake apparently ceased and the total radioactivity in the organisms remained constant (Fig. 3). However, since material was being released into the medium after this time (Fig. 1), it is probable that the synthesis and degradation of peptidoglycan were in equilibrium.

To determine whether the rate of incorporation of meso-diaminopimelic acid remained constant during glycerol-induced myxospore formation, a pulse-labelling experiment was performed. Organisms were exposed to labelled amino acid for $20 \mathrm{~min}$ and the amount of radioactivity incorporated into TCA-insoluble material was determined. At 40 to $50 \mathrm{~min}$ after the addition of glycerol the amount of label incorporated during each pulse doubled, compared with non-induced growing cultures, and was maximal during the shape change associated with myxospore formation (Fig. 4).

\section{Autolytic activity and wall turnover in a glycerol-insensitive mutant}

The results described above indicated that glycerol might affect the activation or induction of autolytic enzymes which result in the increased wall turnover observed. These results did not indicate how relevant these effects are to the formation of myxospores. It seemed pertinent, therefore, to investigate a mutant that could not be induced to form myxospores by glycerol.

Kottel \& White (1974) have described an increase in autolytic activity occurring during myxospore formation. We have confirmed this observation (Fig. 5). When glycerol was added to the non-inducible mutant no increase in autolysis was observed (Fig. 5), clearly indicating that cellular morphogenesis and autolytic activity are associated. Furthermore, when the rate of wall turnover in the mutant was measured in the presence and absence of glycerol it was observed that vegetative organisms had a low rate of turnover similar to the parent strain, but this was increased slightly by glycerol (Fig. 6).

\section{Wall turnover in the presence of phenethyl alcohol}

Phenethyl alcohol induced myxospore formation in both the parent and glycerolinsensitive strains. Induction with phenethyl alcohol, in contrast to glycerol, was asynchronous and the first rounded cells were not apparent until $3 \mathrm{~h}$ after adding the inducer. A 


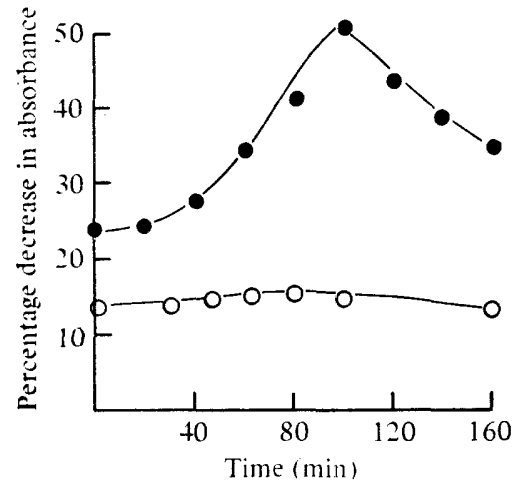

Fig. 5

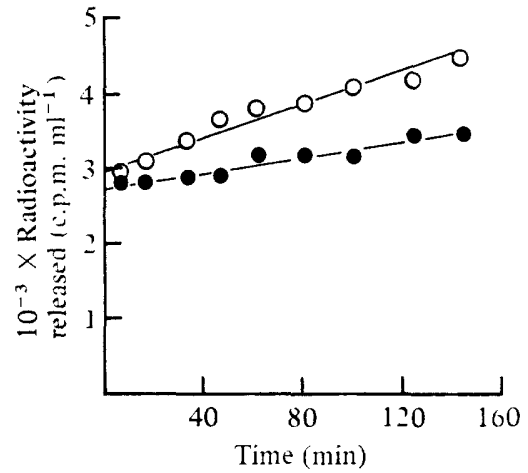

Fig. 6

Fig. 5. Autolytic activity during myxospore induction in Myxococcus xanthus. Glycerol ( $0.5 \mathrm{M})$ was added to the parent $(\mathcal{O})$ and glycerol-insensitive mutant $(O)$ strains growing in $1 \%$ Casitone medium. Samples were transferred to distilled water at $50^{\circ} \mathrm{C}$ and the percentage decrease in absorbance during a 15 min period was determined.

Fig. 6. Meso-diamino $\left[{ }^{14} \mathrm{C}\right]$ pimelic acid release by a glycerol-insensitive mutant of Myxococcus xanthus. Prelabelled organisms were incubated in $1 \%$ Casitone medium with $(O)$ or without $(O)$ $0.5 \mathrm{M}$-glycerol. Radioactivity released was measured in the supernatant after removal of the organisms by centrifugation.

large proportion of the treated organisms (up to $50 \%$ ) always failed to respond to phenethyl alcohol and most of these eventually lysed.

Measurements of the release of meso-diaminopimelic acid from prelabelled organisms, after the addition of phenethyl alcohol, showed that there were slight increases in turnover in both the parent and mutant strains. Increases in rates of release from 1.5 to $3 \% \mathrm{~h}^{-1}$ to 2.5 to $5.5 \% \mathrm{~h}^{-1}$ on addition of phenethyl alcohol were observed. These were considerably less than those observed when glycerol was added to the parent strain.

\section{DISCUSSION}

Previous studies with developing myxospores have shown that vegetative organisms and myxospores possess the same amounts of peptidoglycan (White et al., 1968) but that myxospore peptidoglycan is more cross-linked (Johnson \& White, 1972). Additionally, during the glycerol-induced formation of myxospores their walls become more prone to autolysis (Kottel \& White, 1974) and show increased sensitivity to some antibiotics (White et al., 1968). These studies did not reveal to what extent vegetative wall material is retained by the developing myxospore or the role of the inducer, glycerol, in bringing about these changes.

Our results have shown that, following the addition of glycerol, there is both an extensive loss (Fig. 1) and a greatly increased rate of incorporation (Fig. 4) of the specific peptidoglycan amino acid, meso-diaminopimelic acid. This very high level of turnover must result in substantial replacement of existing wall with newly synthesized material. It is difficult to quantify exactly the degree to which the existing peptidoglycan is degraded since recently synthesized material is more susceptible to autolysis (H. Dawson \& M. V. Jones, unpublished results). Similar differences in susceptibility have also been found in Streptococcus faecalis (Shockman et al., 1967).

What is the mechanism by which glycerol induces myxospore formation? The change in cell shape occurs in less than one generation time and is synchronous. This means that the initial events, at least, are independent of the cell-cycle, although subsequently the failure of organisms to initiate new rounds of DNA replication (Rosenberg et al., 1967) will bring 
them into step. Additionally, glycerol and other inducers need not penetrate the cytoplasmic membrane to have an effect (Sadler \& Dworkin, 1966). These data strongly implicate the envelope as the primary site of inducer action and we would suggest that glycerol interacts directly with peptidoglycan degradation and synthesis. Activation of autolytic enzymes by glycerol was first proposed by White et al. (1968), and we have now obtained some experimental evidence to support this theory. An increase in the rate of release of meso-diaminopimelic acid is observed on adding glycerol to a growing culture (Figs 1,2), and this precedes the increased incorporation of meso-diaminopimelic acid (Fig. 4). This increased rate is observed even when protein synthesis is inhibited by chloramphenicol. However, a second increase in the rate of amino acid release about $40 \mathrm{~min}$ after adding glycerol is reduced by chloramphenicol, and cellular morphogenesis is not observed. This can be interpreted as meaning that, although glycerol addition results in activation of some autolytic enzymes already present within the envelope, subsequent synthesis of more enzyme(s) is necessary for the shape change to occur. Whether glycerol itself or a product of the 'activated' autolytic enzyme is the inducer is uncertain at this time. The results obtained with the glycerolinsensitive mutant give further support to the proposal that changes in wall turnover are important in glycerol-induced cellular morphogenesis. The mutant neither formed myxospores in the presence of glycerol nor displayed increased autolytic activity as measured by cell lysis (Fig. 5) or release of meso-diaminopimelic acid (Fig. 6). The mutant could be induced by phenethyl alcohol but, like the parent strain, did not show substantially increased rates of meso-diaminopimelic acid release.

Other glycerol-insensitive mutants have also been found to respond to phenethyl alcohol (Burchard \& Parish, 1975) and this observation has led to the proposal that the two inducers have different modes of action. Our data on wall turnover lend support to this suggestion.

The results described concern the release and incorporation of meso-diaminopimelic acid. Although this amino acid comprises part of the peptidoglycan molecule, these results do not indicate to what extent the peptidoglycan is completely degraded. Such information is necessary if we are to recognize the types of autolytic enzymes involved and to understand the role of glycerol in the control of their activity.

We thank Professor H. R. Perkins for his advice and encouragement during the course of this work. We also thank the S.R.C. for a studentship (to Hilary Dawson).

\section{REFERENCES}

Burchard, R. P. \& Parish, J. H. (1975). Mutants of Myxococcus xanthus insensitive to glycerolinduced myxospore formation. Archives of Microbiology 104, 289-292.

Dawson, H. \& Jones, M. V. (1978). Peptidoglycan turnover during myxospore formation and germination in Myxococcus xanthus. Proceedings of the Society for General Microbiology 5, 53.

Dworkin, M. \& Gibson, S. M. (1964). A system for studying microbial morphogenesis: rapid formation of microcysts in Myxococcus xanthus. Science 146, 243-244.

Johnson, R. Y. \& White, D. (1972). Myxospore formation in Myxococcus xanthus: chemical changes in the cell wall during cellular morphogenesis. Journal of Bacteriology 112, 849-855.

KotTel, R. \& WhiTe, D. (1974). Autolytic activity associated with myxospore formation in Myxococcus xanthus. Archives of Microbiology 95, 91-95.
Rosenberg, E., Katarski, M. \& Gottlieb, P. (1967). Deoxyribonucleic acid synthesis during exponential growth and microcyst formation in Myxococcus xanthus. Journal of Bacteriology 93, 1402-1408.

SADLER, W. \& DWORKIN, M. (1966). Induction of cellular morphogenesis in Myxococcus xanthus. II. Macromolecular synthesis and mechanism of inducer action. Journal of Bacteriology 91, 15201525.

Shockman, G. D., Pooley, H. M. \& Thompson, J. S. (1967). Autolytic enzyme system of Streptococcus faecalis. III. Localization of the autolysin at the sites of cell wall synthesis. Journal of Bacteriology 94, 1525-1530.

White, D., DWorkin, M. \& Tipper, D. J. (1968). Peptidoglycan of Myxococcus xanthus: structure and relation to morphogenesis. Journal of Bacteriology 95, 2186-2197. 